\title{
Valoración de las necesidades de cuidados del paciente durante la hemodiálisis y su relación con el grado de dependencia
}

\author{
María del Carmen López Toledano, Isabel Luque Cantarero, Victoria Eugenia Gómez López, Rafael Casas \\ Cuesta
}

U.G.C. Nefrología. Hospital Universitario Reina Sofía de Córdoba

\section{Resumen}

Introducción: En la actualidad, el paciente en hemodiálisis tiene más edad, comorbilidad añadida y algún grado de dependencia. Esta situación ha hecho que su atención en las unidades de diálisis sea cada más compleja, relacionándose a menudo grado de dependencia con mayor necesidad de cuidados de enfermería. Sin embargo, no existen estudios donde se evidencie este hecho de forma concreta.

Objetivo: El objetivo del presente estudio fue evaluar el grado de dependencia de los pacientes en hemodiálisis y su relación con las necesidades de cuidados de enfermería durante la sesión.

Pacientes y Métodos: Se estudiaron 88 pacientes en hemodiálisis, del Servicio de Nefrología del HURS de Córdoba, mediante un diseño observacional analítico. Para evaluar el grado de dependencia se utilizó el "Test Delta", y el "Índice de Katz". Para analizar la demanda de cuidados se usó una parrilla de actividades de enfermería durante la sesión, de elaboración propia.

Resultados: La edad media fue 64,87 años y en el índice de comorbilidad se encontró una mediana de 7 (rango $0-12$ ). Cuando se analizaron los resultados del Test Delta, comprobamos que el $69,3 \%$ presentaban algún tipo de dependencia, mientras que con el Índice de Katz la presentaban el $72,7 \%$, encontrándose entre ambos una buena correlación $(r=0,866 p<0,01)$. Se encontró correlación entre la edad y las actividades de enfermería $(r=0,331, p<0,01)$, el índice de comorbilidad $(r=0,726$, $p<0,01)$ y el Test Delta $(r=0,244, p<0,05)$. También se encontró correlación entre las actividades de enfermería

\section{Correspondencia:}

Rafael Casas Cuesta

UGC Nefrología. Hospital Universitario Reina Sofía

Avda. Menéndez Pidal, s/n. 14005 Córdoba

E-mail: rafcasas@ono.com y el Delta, $(r=0,483, p<0,05)$, el Katz $(r=0,405 p<0,01)$ y el índice de comorbilidad $(r=0,380 p<0,01)$. A su vez el índice de comorbilidad se correlacionó con el Delta $(r=0,320 p<0,05)$ y el índice de Katz $(r=0,276 p<0,01)$.

Conclusiones: Nuestros resultados sugieren que a mayor grado de dependencia, indistintamente del instrumento utilizado, mayor necesidad de cuidados. De igual manera, a más edad y mayor índice de comorbilidad, más necesidad de cuidados de enfermería durante la sesión de hemodiálisis. Sería necesario profundizar en el estudio de la parrilla de cuidados durante la sesión.

PALABRAS CLAVE

- GRADO DE DEPENDENCIA

- HEMODIÁLISIS

- CUIDADOS ENFERMERÍA

- CARGA DE TRABAJO ENFERMERÍA

- COMORBILIDAD

Assessment of patient care needs during hemodialysis and its relationship with the level of dependence

\section{Abstract}

Introduction: Currently, haemodialysis patients are older, higher comorbidity added and a certain greater degree of dependence. This situation causes that the attention in dialysis units become increasingly complex, often related to the degree of dependence with higher patient care needs. However, there are no studies where this fact is evidenced concretely.

Objective: The aim of this study was to evaluate the dependence of haemodialysis patients and their relationship to the needs of nursing care during the session. 
Patients and Methods: 88 haemodialysis patients were studied in the Nephrology Unit of the HURS of Córdoba, through an analytical observational design. To assess the degree of dependence, we used the "Delta Test" and the "Katz index". To analyse the demand for care, we used during the session, a homemade table of nursing activities.

Results: The mean age was 64.87 years, with a mean comorbidity index of 7 (range $0-12$. When Delta Test results were analyzed, we found that $69.3 \%$ had some type of dependency, while the Katz index showed $72.7 \%$, finding a good correlation between the two measures $(r=0.866 ; p<0.01)$. Correlations between age and nursing activities ( $r=0.331 ; p<0.01)$, comorbidity index $(r=0.726 ; p<0.01)$ and the Delta Test $(r=0.244 ; p<0.05)$ were found.

Conclusions: Our results suggest that the greater the degree of dependence, regardless of the instrument used, the greater care needs. Similarly, the higher age and comorbidity, higher need for nursing care during haemodialysis. It would be necessary deepen on the study of the table care during the session.

\section{KEYWORDS}

- DEGREE OF DEPENDENCY

- HAEMODIALYSIS

- NURSING CARE

- NURSING WORKLOAD

- COMORBIDITY

\section{Introducción}

La Enfermedad Renal Crónica Avanzada (ERCA) ha aumentado su prevalencia considerablemente en la última década, y con ello la cifra de pacientes sometidos a tratamiento sustitutivo de la función renal. Además del envejecimiento progresivo de la población, el aumento de la prevalencia de otros procesos crónicos como la diabetes mellitus, hipertensión y obesidad, contribuyen sin lugar a dudas, al aumento del número de pacientes que reciben tratamiento sustitutivo de la función renal, a pesar de que los últimos datos señalan que la incidencia va estabilizándose $e^{1,2}$.

Según el último registro de la Sociedad Española de Nefrología ${ }^{3}$, la demanda de tratamientos sustitutivo va creciendo de forma significativa. Este informe señala como principal causa de ERCA en mayores de 15 años, con un $24,91 \%$ la diabetes mellitus, siendo las patologías cardiovasculares la principal causa con un $45 \%$ en mayores de 75 años. Tanto los pacientes sometidos a tratamiento sustitutivo como los trasplantados, la supervivencia es mayor en el grupo de menos edad. La incidencia aumenta en función de la edad, los pacientes comprendidos entre 45-64 años, representan el 33\% de la población total. Del mismo modo, la prevalencia es mayoritaria en el grupo de pacientes de edades comprendidas entre 45-64 años con un 39\%. El tratamiento de elección es la hemodiálisis (HD) seguido del trasplante renal. Este dato está estrechamente relacionado con la edad del paciente, ya que la HD es el tratamiento más utilizado por los pacientes mayores de 75 años, seguido por el grupo de pacientes entre 65-74 años. Hay que señalar, que un porcentaje importante de pacientes mayores, no se pueden trasplantar, por lo que solo tienen la opción dialítica para poder vivir ${ }^{3,4}$.

Por tanto, la ERCA constituye un problema de salud a nivel global, caracterizado por la creciente incidencia y prevalencia en la población general ${ }^{5}$. Esto va a determinar mayor demanda de tratamiento sustitutivo de la función renal, discapacidades asociadas, disminución de la calidad de vida, prematura mortalidad y un elevado consumo de servicios sanitarios por parte de estos pacientes ${ }^{5,6,7}$.

Diferentes estudios, muestran que la ERCA así como la diálisis, afectan a las distintas áreas del paciente, desde su salud física a la psicológica, especialmente a nivel emocional y cognitivo y social, lo que va a influir en su calidad de vida, ${ }^{8,9}$; siendo cada vez mayor el número de pacientes que se dializan con alguna discapacidad ${ }^{10}$. Esta dependencia funcional, obliga a una adaptación de los cuidados por parte de los profesionales a esta circunstancia individual; por ello se deben utilizar los instrumentos necesarios para medir y conocer las necesidades de cada paciente. En efecto, la edad y la comorbilidad asociada de la mayoría de los pacientes en HD dificultan su autonomía funcional, requiriendo un mayor número de cuidados para poder cubrir sus necesidades ${ }^{11,12,13}$.

La mayor parte de los cuidados diarios que requiere la población objeto de nuestro estudio, recae sobre los denominados cuidadores informales, entre los que podemos destacar familiares directos en la mayoría de los $\operatorname{casos}^{14}$. La labor del cuidador principal abarca desde cubrir las necesidades del paciente, manteniendo a este en su entorno social, disminuyendo la utilización de recursos formales y evitando la institucionalización del paciente $\mathrm{e}^{15,16,17}$.

Por todo ello, hay que tener en cuenta la necesidad de cuidados que plantean hoy día los pacientes durante 
la HD, para poder garantizar que estas se encuentren cubiertas. Existe en el momento presente un interrogante, no suficientemente contestado, en el sentido de si los actuales ratios pacientes/enfermera de las unidades de diálisis debe variar ${ }^{18}$, teniendo en cuenta que el perfil del paciente atendido en las unidades de diálisis ha sufrido un cambio en los últimos tiempos, relacionado con el grado de dependencia de estos, y agravadas en algunos casos por la edad o pluripatología, pues existen estudios que han asociado un mayor grado de dependencia con mayor necesidad de cuidados, aunque no está establecida una relación estadística ${ }^{19}$.

Es por esto, que debemos cuestionarnos si el aumento del grado de dependencia de los pacientes es proporcional a la necesidad de cuidados, con el consiguiente aumento de personal de enfermería, o si por el contrario este hecho no influye tanto en la necesidad de cuidados ${ }^{19,20}$, por lo que queremos realizar este estudio, profundizando no solo en las necesidades para la vida diaria que plantean los pacientes, sino concretando los cuidados que precisan durante su estancia en la unidad de diálisis.

Por tanto, el objetivo de este estudio fue evaluar el grado de dependencia de pacientes en HD y su relación con las necesidades de cuidados de enfermería durante la sesión.

\section{Pacientes y métodos}

\section{Pacientes}

Se realizó un estudio observacional analítico, en el Servicio de Nefrología del Hospital Universitario Reina Sofía de Córdoba.

La población de estudio fueron los pacientes en HD de la provincia de Córdoba. Como muestra se eligió a todos los pacientes que se dializaban en la Unidad de Diálisis Hospitalaria y en el Centro Periférico de Diálisis, por ser a los pacientes que tuvimos acceso. Se estudiaron un total de 88 pacientes. Se excluyeron a 5 pacientes que no quisieron participar en el estudio.

\section{Variables del estudio}

Las variables utilizadas para el estudio fueron:

- Edad y sexo.

- Tiempo medio en HD.

- Centro de diálisis.

- Comorbilidad.

- Nivel de dependencia.

- Necesidades de cuidados de enfermería.

\section{Instrumentos para la recogida de datos}

Para la evaluación del grado dependencia se utilizó el "Índice de Katz", que mide las actividades de la vida diaria. Es un instrumento validado y fácil de cumplimentar ${ }^{21}$. Este índice consta de 6 ítems, con tan solo dos posibles respuestas, dependiente 0 independiente.

Se realizó también el análisis de dependencia mediante el "Test Delta", por ser este un cuestionario validado para pacientes en $H D$, y que recoge aspectos concretos de la estancia del paciente en la unidad de diálisis ${ }^{22,23}$. El "Test Delta" consta de 3 dimensiones: A. Dependencia en general, B. Deficiencia física y C. Deficiencia psíquica. El resultado del test Delta será la suma de las 3 áreas, donde las puntuaciones máximas son: Deficiencia general: puntuación máxima 30 puntos. Deficiencia física: puntuación máxima 15. Deficiencia psíquica: puntuación máxima 15.

Para conocer la comorbilidad de los pacientes, se utilizó el "Índice de Comorbilidad de Charlson" (ICC)24,25. Este índice recoge los componentes por enfermedades asociadas, relacionándolo con la edad.

Para analizar las necesidades de cuidados de enfermería durante la sesión de HD, se elaboró una parrilla de necesidades que los pacientes pueden presentar antes, durante y después de la sesión de diálisis. Esta parrilla, se elaboró a partir del consenso de un grupo de enfermeras expertas, que elaboraron un listado con estas actividades y posteriormente las ponderaron y consensuaron, en función del tiempo y dedicación que ocupa cada una de ellas. A este cuestionario lo hemos denominado "actividades enfermeras durante la HD" (AEDHD). Estos cuestionarios fueron cumplimentados por el personal de enfermería de la propia unidad, dado su conocimiento de los pacientes. La mínima puntuación significa menos necesidad de cuidados y la máxima mayor necesidad de cuidados. Los ítems y la valoración de estos, podemos encontrarla en el Anexo I.

Todos los participantes fueron informados de la naturaleza y los objetivos del estudio, solicitándosele en ese momento el consentimiento verbal.

\section{Recogida de datos}

Para garantizar confidencialidad de los datos de los pacientes, las entrevistas se realizaron por un único investigador y sin tener acceso a la base de datos del propio servicio. Las encuestas iban numeradas por el investigador principal, con las que posteriormente realice mi propia base de datos. Posteriormente el in- 
vestigador principal introdujo en la tabla de datos las variables propias de la base de datos del servicio (edad, tiempo HD, ICC), garantizándose en todo momento la confidencialidad de los datos y anonimato de los pacientes.

\section{Análisis estadístico}

Se ha realizado una distribución de frecuencias para el análisis de las variables cualitativas y para las variables numéricas se ha calculado la media \pm la desviación estándar, y la mediana cuando no seguían una distribución normal. Para la relación entre variables se ha utilizado el coeficiente de correlación Pearson. Se aceptó significación estadística para $p<0.05$. El análisis estadístico se realizó con el paquete informático SPSS 15.0.

\section{Resultados}

Se estudiaron 53 hombres $(60,2 \%)$ y 35 mujeres $(39,8 \%$ ), con una edad media de 64,87 $\pm 17,588$ (rango 22-97). El 35,2\% se dializaban en el Hospital Reina Sofía, mientras el 64, 8\% lo hacen en el Centro Periférico de diálisis. El tiempo medio de HD fue de $62,39 \pm 61,39$ meses (rango 2-325).

Cuando se analizaron los resultados globales del Test Delta, obtuvimos un valor medio de 7,10 $\pm 8,3$ y una mediana de 4 (rango 0-34). Encontramos que el 69,3\% de los pacientes presentaban algún tipo de dependencia, frente a un $30,7 \%$ que fueron considerados válidos. En la Figura 1, se distribuyen los pacientes según el grado de dependencia.

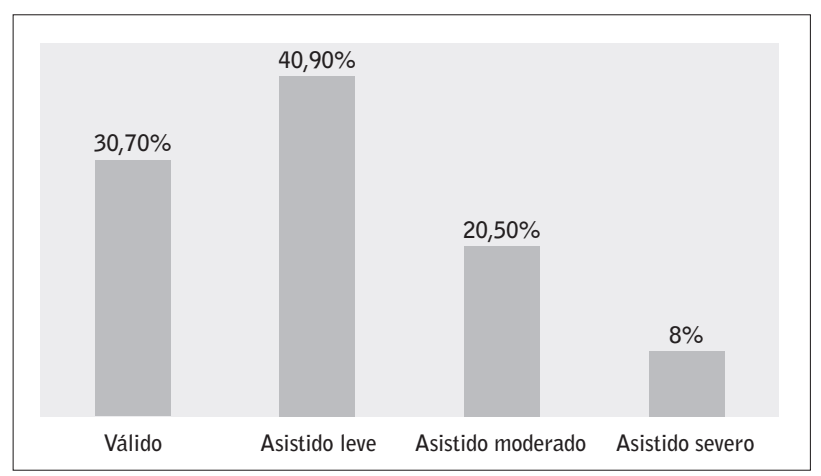

Figura 1. Resultados Test Delta por grado de dependencia.
En la Figura 2, se representa dentro de los 4 grados de dependencia, la distribución de pacientes en cada una de las dimensiones o áreas que evalúa el Test Delta.



Figura 2. Distribución de pacientes en cada una de las dimensiones del Test Delta.

Cuando se analizó la dependencia con el índice de Katz, el valor medio fue de $0,82 \pm 1,6$ y una mediana de 0 (rango 0-7), siendo el $72,7 \%$ totalmente independiente en todos los ítems del cuestionario, presentando el $27 \%$ algún tipo de dificultad o dependencia para desarrollar las actividades de la vida diaria.

Tras estudiar los datos obtenidos del ICC, con una mediana de 7 (rango 0-12), podemos comprobar que el $94,4 \%$ de los pacientes presentaron una comorbilidad alta, tal como muestra la Figura 3.

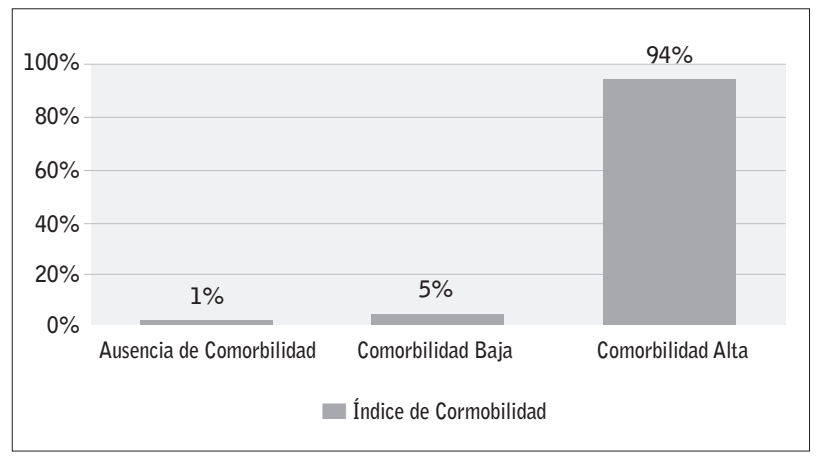

Figura 3. Distribución del ICC, según grado de comorbilidad.

Con respecto AEDHD, se encontró una mediana de 8 (rango 3.-20), oscilando el mayor porcentaje entre puntuaciones de 5-11, expresado en la Figura 4. 


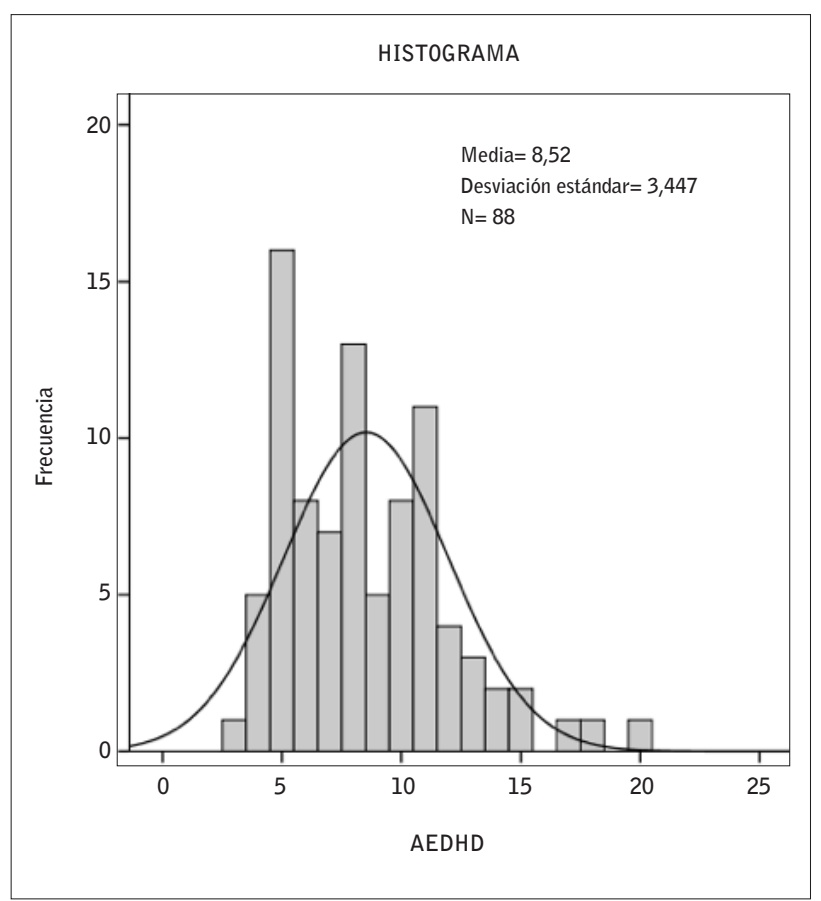

Figura 4. Puntuación de AEDHD.

Cuando se relacionaron las distintas variables, encontramos correlación significativa entre la edad y las $\operatorname{AEDHD}(r=0,331, p<0,01)$, el ICC $(r=0,726, p<0,01)$ y el Test Delta $(r=0,244, p<0,05)$.

También se encontró correlación estadísticamente significativa entre el Delta con AEDHD, $(r=0,483, p<0,05)$ $y$ con el Centro $(r=-0,394 p<0,01$.). Entre ICC y AEDHD $(r=0,380 p<0,01)$ y el Delta $(r=0,320 p<0,05)$.

De igual forma, se encontró correlación significativa del índice de Katz con AEDHD $(r=0,405 p<0,01)$, con Delta $(r=0,866 p<0,0) 1$ y IC $(r=0,276 p<0,01)$, y el Centro $(r=-0,440 p<0,01)$.

No se ha encontrado ninguna otra relación significativa entre el resto de variables.

\section{Discusión}

El cambio en el perfil del paciente con patología renal ha cambiado en los últimos años y con ello las necesidades de cuidados, generando esto preocupación en los profesionales de enfermería ${ }^{13}$. Este hecho viene muy determinado por el aumento de edad poblacional del paciente en HD, pues la mayoría de los pacientes, son mayores de 65 años 3,26 .

Son variados los instrumentos de los que se disponen en la actualidad para valorar el grado de dependencia, pero todos están diseñados para evaluar las actividades de la vida diaria. El test Delta, sin embargo, se adaptó y validó para evaluar el grado de dependencia del paciente en HD.

En nuestro estudio, cuando analizamos el grado de dependencia con el test Delta, encontramos que más de la mitad de los pacientes presentaban dependencia en algún grado (69,3\%). A analizar las distintas dimensiones del test Delta los pacientes presentan mayor dependencia en las dimensiones de déficit general, seguido de déficit físico, siendo destacable que el 40,9\% de los pacientes, son asistidos leves. La mayoría de los estudios analizados coinciden con nuestros resultados, encontrando mayor puntuación en los aspectos relacionados con la movilidad 22,23 .

Con respecto al índice de Katz, solo un $27,3 \%$ de la muestra presenta dependencia. Esto puede ser debido a que tan solo evalúa 6 dimensiones de las actividades de la vida diaria, no existiendo estudios previos que utilicen este índice en pacientes en HD. Sin embargo, en nuestro estudio presenta una buena correlación con Delta, con el ICC y con el AEDHD. Este hecho, es digno de resaltar, teniendo en cuenta de que en el momento de realizar las entrevistas, es un cuestionario muy sencillo.

Se han utilizado, diferentes instrumentos para medir el grado de dependencia, pero los resultados pueden variar para un mismo individuo. Algunos de estos cuestionarios, también muy utilizados, son la Valoración Física de Lawton, el Índice de Barthel (IB), o el Baremo de la Ley de Dependencia ${ }^{27}$, al igual que otros cuestionarios propios creados para el estudio en cuestión. Normalmente en estos estudios no suelen utilizarse de forma aislada, sino por el contrario se asocian dos o más, con el fin de hacer comparativas y poder abarcar la dependencia de los pacientes en todas sus dimensiones, de ahí que en nuestro estudio nos decidiéramos por utilizar dos instrumentos.

Otro aspecto importante, en el análisis de la dependencia del paciente en HD, es su comorbilidad, pues se ha asociado a edad y dependencia con el test Delta ${ }^{22}$, pues la mayor parte de los pacientes presentan comorbilidad alta, condicionando esta el grado de dependencia de forma significativa. En nuestros pacientes, también se encontró una estrecha relación entre el ICC y Delta, estando a su vez, estas variables relacionadas estadísticamente con la edad.

Al comienzo de nuestro estudio nos planteamos si la dependencia, medida con los instrumentos habituales, era un buen predictor de las necesidades de cuidados de enfermería durante la sesión de diálisis, pues 
estudios previos habían asumido que por el hecho de ser más dependientes, los pacientes presentaban más necesidades de cuidados de enfermería, y/o necesidades de tiempo de enfermería ${ }^{18,19}$. De ahí, la elaboración de la parrilla de cuidados específicos durante la HD. Pues bien, cuando relacionamos las AEDHD, con el resto de variables, pudimos observar cómo se correlacionó de manera estadísticamente significativa con ambos instrumentos de medida del grado de dependencia (Delta y Katz), comorbilidad y edad del paciente. Estos resultados, además de coherentes, son lógicos; pues como ha quedado expresado anteriormente, estas tres variables se relacionan estrechamente entre si, en los estudios previos. De igual forma, la parrilla de elaboración propia, aunque no está validada, presenta consistencia a la hora de valorar las necesidades de los pacientes, al correlacionarse de forma significativa con dependencia, comorbilidad y edad. En cuanto, a los tiempos dedicados al cuidado durante la sesión, observamos que la mayor frecuencia es para la dificultad en la punción de la fístula arteriovenosa interna y control de constantes por inestabilidad hemodinámica, con frecuentes episodios de hipotensión, los cuales requieren administración de medicación endovenosa.

Sin embargo, algunos autores, comunicaron que el tiempo de enfermería dedicado al paciente durante la $H D$, es independiente del grado de dependencia o del aumento del envejecimiento, estando más asociado a variables, como el tipo de acceso vascular ${ }^{19}$.

Por todo esto, pensamos que es fundamental a la hora del debate de los tiempos de enfermería dedicados al cuidado del paciente en HD y su relación con las ratio enfermera/pacientes, que este se centre en analizar realmente la demanda de cuidados en la unidad de diálisis. Aunque hay estudios, que además asociar la percepción de enfermería y el nivel de dependencia de los pacientes, afirman la existencia de otros factores que van a intervenir en la carga de trabajo de la enfermera en HD, no han documentado la posibilidad de calcular la asignación enfermera/paciente por este hecho ${ }^{28}$. Es imprescindible, por tanto, la validación de instrumentos propios para medir la necesidad de cuidados de enfermería durante la sesión de diálisis.

Las limitaciones del presente estudio vienen determinadas por la imposibilidad de realizar muestreo, pues solo se tuvo acceso a la muestra de estudio, y en segundo lugar a que la parrilla de cuidados elaborada por el grupo de enfermería del Servicio de Nefrología, no está validada, y necesita de mas estudios intentar que sea un instrumento útil y fácil de manejar.
A la vista de nuestros resultados podemos afirmar, al menos en nuestros pacientes, que la mayoría de ellos presentan en mayor o menor medida, algún grado de dependencia. Esta dependencia tiene además, relación significativa con el aumento de la edad y la comorbilidad asociada.

Es necesario profundizar en la medida de la necesidad de cuidados de enfermería del paciente en HD, durante su estancia en la unidad de diálisis, pues como hemos visto la edad, comorbilidad y dependencia se asocian significativamente con los cuidados realizados a los pacientes durante la sesión.

Recibido: 2 septiembre 2014

Revisado: 20 septiembre 2014

Modificado: 26 septiembre 2014

Aceptado: 27 septiembre 2014

\section{Bibliografía}

1. Luño J, García De Vinuesa S, Gómez-Campderá F, Et Al. Factores predictivos en la progresión de La enfermedad renal. Nefrología. 1999; 19(6):524530.

2. De Francisco A, De la Cruz J, Cases A, Et Al. Prevalencia de insuficiencia renal en centros de atención primaria en España: Estudio Erocap. Nefrología. 2007; 27(3):300-312.

3. Informe de diálisis y trasplantes. XIII Congreso Nacional de la Sociedad Española de Nefrología. 2012.

4. Heras,M., García-Cosmes,P., Fernández-Reyes, M. J., Sánchez, R. Evolución natural de la función renal en el anciano: análisis de factores de mal pronóstico asociados a la enfermedad renal crónica. Nefrología 2013; 33(4): 462-9.

5. Valderrábano F. El tratamiento sustitutivo de la insuficiencia renal crónica en España. Nefrología. 1994; 14(Supl 1):27-35

6. García Pérez R. Pacientes geriátricos en hemodiálisis. Diálisis del anciano. Rev Soc Esp Enferm Nefrol. 2001; 15: 64-73

7. Treviño-Becerra, A. Insuficiencia Renal Crónica: Enfermedad emergente, catastrófica y por ello prioritaria: Cir. Ciruj. 2004; (72): 3-4. 
8. Alcázar $R$, De Francisco A. Acción estratégica de la SEN frente a la enfermedad renal. Nefrología. 2006; 26:1-4.

9. Álvarez-Ude, F. Factores asociados al estado de salud percibido (calidad de vida relacionada con la salud) de los pacientes en hemodiálisis crónica. Rev Soc Esp Enferm Nefrol. 2001; 4 (2): 64-68.

10. Álvarez-Ude F, Fernández-Reyes $M$, Vázquez $A$, Mon C, Sánchez R, Rebollo P. Síntomas físicos y trastornos emocionales en pacientes en programa de hemodiálisis periódicas. Nefrología. 2001; 21(2):191-199.

11. Saracho-Rotaeche R. El afrontamiento como predictor de la calidad de vida en diálisis: Un estudio longitudinal y multicéntrico. Nefrología. 2013; 33(3):342-354.

12. Andreu Periz L, Moreno Arroyo Mc. Perfil y realidad social de los cuidadores principales de pacientes dependientes tratados con hemodiálisis. Nursing. 2009; (27): 54-65.

13. Contreras Abad MD, Rivero Arellano MF, Jurado Torres MJ, Crespo Montero R. Perfil actual del paciente en hemodiálisis hospitalaria: Análisis de sus necesidades. Rev Soc Esp Enferm Nefrol. 2004; 7(1):56-61.

14. Andreu Periz L, Moreno Arroyo C, Julve Ibañéz M. Valoración de pacientes tratados con hemodiálisis según la Ley de promoción de la autonomía personal y atención a las personas en situación de dependencia. Rev Soc Esp Enferm Nefrol. 2010; 13(4):252-257.

15. Aladrén $M$, Pérez $J$, Azuara $M$, Berisa $F$. Hemodiálisis en pacientes de edad avanzada. Estudio multicéntrico de las sociedades Aragonesa Y Norte de Nefrología. Nefrología. 1999; 19(1).

16. González A. Guías SEN para el manejo de la enfermedad renal crónica avanzada y prediálisis. Nefrología. 2008; (Supl 3):1.

17. Gila M, Sánchez R, Gómez-Caro S, Oropesa A, Morena J, Moreno F. El rol de cuidador de personas dependientes y sus repercusiones sobre su calidad de vida y su salud. Revista Clínica de Medicina Familiar. 2009; 2(7):332-334.

18. González A, Rivera J, Novoa S, Et al. Efectividad de la intervención educativa en cuidadores de pacientes dependientes en diálisis y valoración de la carga. Rev Soc Esp Enferm Nefrol. 2005; 8(2).

19. Cobo Sánchez JL, Et al. Repercusión del grado de dependencia de los pacientes en hemodiálisis sobre la carga de trabajo de enfermería. Rev Soc Esp Enferm Nefrol. 2008; (11): 6-11.

20. Barrios Araya S, Arechabala Mantuliz MC, Valenzuela Parada V. Relación entre carga laboral y Burnout en enfermeras de unidades de diálisis. Enfermería Nefrológica. 2012; 15(1):46-55.

21. Valderrama E, Et Al. Una visión crítica de las escalas de valoración funcional traducidas al castellano. Rev Esp Geriatr y Gerontol 1997; 32 (5): 297-306

22. Arribas V, García G, Gil F, Et al. Evaluación del grado de dependencia de los pacientes en hemodiálisis mediante el Test Delta. Vascular.7:12.13.

23. Arenas M, Álvarez-Ude F, Angoso M, Et al. Valoración del grado de dependencia funcional de los pacientes en hemodiálisis: Estudio multicéntrico. Nefrología. 2006; 26(5):600-608.

24. Charlson M, Szatrowski T, Peterson J. Validation of a combined comorbidity Index. J. Clin. Epidemiol 1994; 47(11):1245-1251.

25. Fried L, Ferrucci L, Darer J, Williamson J, Anderson G. Untangling the concepts of disability, frailty, and comorbility: Implications for improved targeting and care. Journal of Gerontology Series A: Biological sciences and medical sciences. 2004; 59(3):M255-M263.

26. Nissenson Ar: Dialysis therapy in the elderly patient. Kidney Int 43.1993; (Supl. 40): S51-S57

27. Camps Ballester E, Andreu Periz L, Colomer Codinachs M, ClaramuntFonts L, Pasaron Alonso M. Valoración del grado de autonomía funcional de pacientes renales crónicos según índices de Barthel, Lawton y baremo de Ley de dependencia. Rev Soc Esp Enferm Nefrol. 2009; 12(2):28-34.

28. Llinás Vidal M. Personal de enfermería en unidades de hemodiálisis frente a personal de enfermería en servicios de hospitalización: Análisis comparativo de personalidad. Comunicaciones presentadas al XIV Congreso Nacional de la SEDEN. Soc Esp Enferm Nefrol. Madrid. Acceso[6 Septiembre 2014]. Disponible en: http://www.seden.org 


\section{ITEMS}

\section{A. Entrada en la unidad}
1. Andando
2. Carrito
3. Camilla

\section{B. Acostarse 0 acomodarse en la cama o sillón}
1. Por su propio pie
2. Ayudado por 1-2 sanitarios
3. Con grúa sanitaria

\section{Conexión a la hemodiálisis}
1. FAVI sin dificultad
2. FAVI con dificultad de punción
3. Catéter sin incidencias
4. Catéter dificultad de QB
5. Protocolo de uroquinasa

\section{Cuidados intrasesión de hemodiálisis (incidencias)}

1. Determinación de glucosa en sangre

1- Tratamiento con oxigenoterapia

2. Cura de herida quirúrgica

3. Cura de UPP o herida

2- Transfusiones frecuentes

1. Medicación IV intra HD

2. Administración o ayuda de ingesta

2- Tratamiento con drogas vasoactivas

3- Incontinencia fecal (aseo)

\section{E. Complicaciones durante la sesión de HD (incidencias)}

\section{- Inestabilidad Hemodinámica}

2. Mayor frecuencia de controles de constantes

1. Monitorización de ECG y/o Sat de Oxígeno

1. Corrección de hipotensión con suero salino

2. Nauseas, vómitos

\section{- Problemas de acceso vascular}

2. Alarma frecuente por déficit de Qd

1. Recolocación de agujas

\section{F. Salida de la unidad}
1. Por su propio pie
2. Ayudado por 1-2 sanitarios
3. Con grúa sanitaria 